\title{
Insulin therapy in critically ill patients
}

This article was published in the following Dove Press journal:

Vascular Health and Risk Management

30 November 2010

Number of times this article has been viewed

\section{Samer Ellahham}

Samer Ellahham and Associates, Falls Church,VA, USA
Correspondence: Samer Ellahham Samer Ellahham and Associates, 370I South George Mason Drive, Suite CI North, Falls Church, VA 2204I, USA Tel +l 7033799202

Email samerl@ix.netcom.com
Abstract: Hyperglycemia frequently occurs with acute medical illness, especially among patients with cardiovascular disease, and has been linked to increased morbidity and mortality in critically ill patients. Even patients who are normoglycemic can develop hyperglycemia in response to acute metabolic stress. An expanding body of literature describes the benefits of normalizing hyperglycemia with insulin therapy in hospitalized patients. As a result, both the American Diabetes Association and the American College of Endocrinology have developed guidelines for optimal control of hyperglycemia, specifically targeting critically ill, hospitalized patients. Conventional blood glucose values of $140-180 \mathrm{mg} / \mathrm{dL}$ are considered desirable and safely achievable in most patients. More aggressive control to $<110 \mathrm{mg} / \mathrm{dL}$ remains controversial, but has shown benefits in certain patients, such as those in surgical intensive care. Intravenous infusion is often used for initial insulin administration, which can then be transitioned to subcutaneous insulin therapy in those patients who require continued insulin maintenance. This article reviews the data establishing the link between hyperglycemia and its risks of morbidity and mortality, and describes strategies that have proven effective in maintaining glycemic control in high-risk hospitalized patients.

Keywords: hyperglycemia, cardiovascular disease, insulin, intensive care, critical care

\section{Introduction}

Diabetes is a chronic disease that causes elevated blood glucose concentrations and disturbances of carbohydrate and lipid metabolism as a result of compromised pancreatic production of insulin or insulin resistance. ${ }^{1}$ In 2007, diabetes affected 23.6 million people in the US, with an estimated annual cost of $\$ 174$ billion, and type 2 diabetes mellitus accounts for $90 \%-95 \%$ of all diagnosed cases in adults. ${ }^{2}$ Approximately onequarter of affected individuals (5.7 million in the US) are undiagnosed, placing them at risk for complications, such as blindness, kidney failure, neuropathy, myocardial infarction, and stroke. ${ }^{2}$ According to data from the 1999-2000 National Health and Nutrition Examination Survey, even among patients diagnosed with diabetes, only $37 \%$ were treated to the glycosylated hemoglobin $\left(\mathrm{HbA}_{1 \mathrm{c}}\right)$ concentration of $<7.0 \%$ recommended by the American Diabetes Association. ${ }^{3}$

Hyperglycemia is a frequent but underappreciated problem seen in many critically ill patients. For critically ill inpatients, hyperglycemia is considered to require treatment when blood glucose level is persistently $\geq 180 \mathrm{mg} / \mathrm{dL}(>10.0 \mathrm{mmol} / \mathrm{L}) .{ }^{4}$ While hyperglycemia during a critical illness is understood to pose a danger for patients with known diabetes, those most at risk of hyperglycemia are actually patients not previously known to have diabetes, in one study accounting for $12 \%$ of all adults admitted 
to hospital. ${ }^{5}$ In this trial, such individuals suffered greater mortality and a lengthier hospital stay than their known diabetic counterparts. ${ }^{5}$

The glucose status of hospitalized patients was once considered less important than the management of their presenting illness. ${ }^{6,7}$ This changed with the recognition that overall outcomes among hospitalized patients may be negatively affected by uncontrolled hyperglycemia or diabetes. Particularly important is that aggressive treatment of hyperglycemia with insulin can minimize morbidity and mortality, a benefit that can extend up to years beyond the immediate illness. ${ }^{5,8}$ Since the first publication of recommendations for management of inpatient hyperglycemia and diabetes in 2004, 7,9 aggressive diagnosis and treatment of hyperglycemia among critically ill patients has become a priority. Standardized protocols typically use a sequenced combination of intravenous and subcutaneous short-, intermediate-, or long-acting insulin formulations. ${ }^{8,10,11}$ The evidence suggests, however, that not all patients exhibit benefits from an intensive insulin therapy regimen (eg, with target blood glucose $<110 \mathrm{mg} / \mathrm{dL})^{12,13}$ as opposed to a conventional glucose target of $140-180 \mathrm{mg} / \mathrm{dL},{ }^{4}$ with the clearest benefits to date demonstrated for surgical intensive care unit (ICU) patients. ${ }^{14}$ These findings suggest the need for further research to define optimal glycemic targets and hyperglycemia management protocols differentially in specific subpopulations of seriously ill patients.

\section{Hyperglycemia and illness}

Hyperglycemia in the hospital setting may occur from stress, decompensation of type 1 or 2 diabetes, or other diabetes, and either withholding various antihyperglycemic medications or administration of hyperglycemia-inducing agents. ${ }^{15}$ Three types of hospitalized patients with hyperglycemia are typically reported in the research literature: those with a medical history of diabetes (eg, fasting blood glucose $\geq 126 \mathrm{mg} / \mathrm{dL}$, twohour oral glucose tolerance test blood glucose $\geq 200 \mathrm{mg} / \mathrm{dL}$, or $\left.\mathrm{HbA}_{1 \mathrm{c}} \geq 6.5 \%\right)^{15}$ which the patient's clinician has diagnosed previously; unrecognized diabetes, ie, hyperglycemia (fasting blood glucose $\geq 126 \mathrm{mg} / \mathrm{dL}$ or random blood glucose $\geq 200 \mathrm{mg} / \mathrm{dL}$ ) occurring during hospitalization but not recognized as frank diabetes by the hospital clinician, with diabetes diagnosis later confirmed after hospitalization by standard criteria; and stress- or hospital-related hyperglycemia, ie, hyperglycemia (fasting blood glucose $\geq 126 \mathrm{mg} / \mathrm{dL}$ or random blood glucose $\geq 200 \mathrm{mg} / \mathrm{dL}$ ) occurring during hospitalization in previously normoglycemic patients and blood glucose level returning to normal during recovery. ${ }^{4,15}$
The $\mathrm{HbA}_{1 \mathrm{c}}$ level can be used to distinguish patients with previously unrecognized diabetes $\left(\mathrm{HbA}_{1 \mathrm{c}} \geq 6.5 \%\right)$ from those who are normoglycemic but experiencing temporary, stress hyperglycemia $\left(\mathrm{HbA}_{1 \mathrm{c}}<6.5 \%\right){ }^{4}$ In suspected cases of stress hyperglycemia, it is important to conduct followup blood glucose testing in order to confirm a persistent return to normoglycemia postdischarge. While some have argued that stress hyperglycemia signals underlying glucose dysregulation or prediabetes, several reports fail to support this notion, showing that few such patients go on to develop frank diabetes. ${ }^{16,17}$

Not all patients who display hyperglycemia require glucose-lowering therapy. To warrant treatment, hyperglycemia must reach certain thresholds and persist over time. ${ }^{4}$ Based on current consensus guidelines, general inpatient hyperglycemia may warrant insulin therapy only when blood glucose values reach a threshold of $\geq 140 \mathrm{mg} / \mathrm{dL}(7.8 \mathrm{mmol} / \mathrm{L}) ;{ }^{4}$ in critically ill patients, the treatment threshold has been set at $180 \mathrm{mg} / \mathrm{dL}(10.0 \mathrm{mmol} / \mathrm{L}){ }^{4}$

Hyperglycemia has been reported in the settings of trauma, stroke, myocardial infarction, and sepsis. ${ }^{18}$ In a study of 100 patients admitted to a medical ICU (including 51 patients with normal glucose homeostasis at baseline), some degree of hyperglycemia was seen in all but four of these patients. ${ }^{18}$ The prevalence of hyperglycemia occurring during a serious illness is especially apparent among patients with cardiovascular disease. In the Euro Heart Survey, 31\% of 4961 adults referred to a cardiologist for treatment of coronary artery disease were found to have diabetes. ${ }^{19}$ Among 923 patients without known diabetes presenting with acute coronary artery disease, $36 \%$ had impaired glucose tolerance and $22 \%$ had newly diagnosed diabetes. Similarly, a retrospective analysis of nondiabetic patients admitted to hospital for acute myocardial infarction revealed a high prevalence of hyperglycemia (at discharge, 35\% had impaired glucose tolerance and $31 \%$ had unrecognized diabetes), which was associated with a substantially increased risk of inhospital morbidity and mortality. ${ }^{20}$ Patients with higher admission glucose levels were more likely to experience subsequent reinfarction, rehospitalization for congestive heart failure, a major cardiovascular event, or death. ${ }^{20}$ As shown in Figure 1, a similar relationship between elevated blood glucose and poor outcomes, including hospital mortality, has been observed in the broader ICU population. ${ }^{21}$ These investigations have shown that glucose levels are an independent predictor of poor short- and long-term outcomes, including mortality, and they suggest that glucose-lowering interventions may improve such outcomes. ${ }^{20}$ 


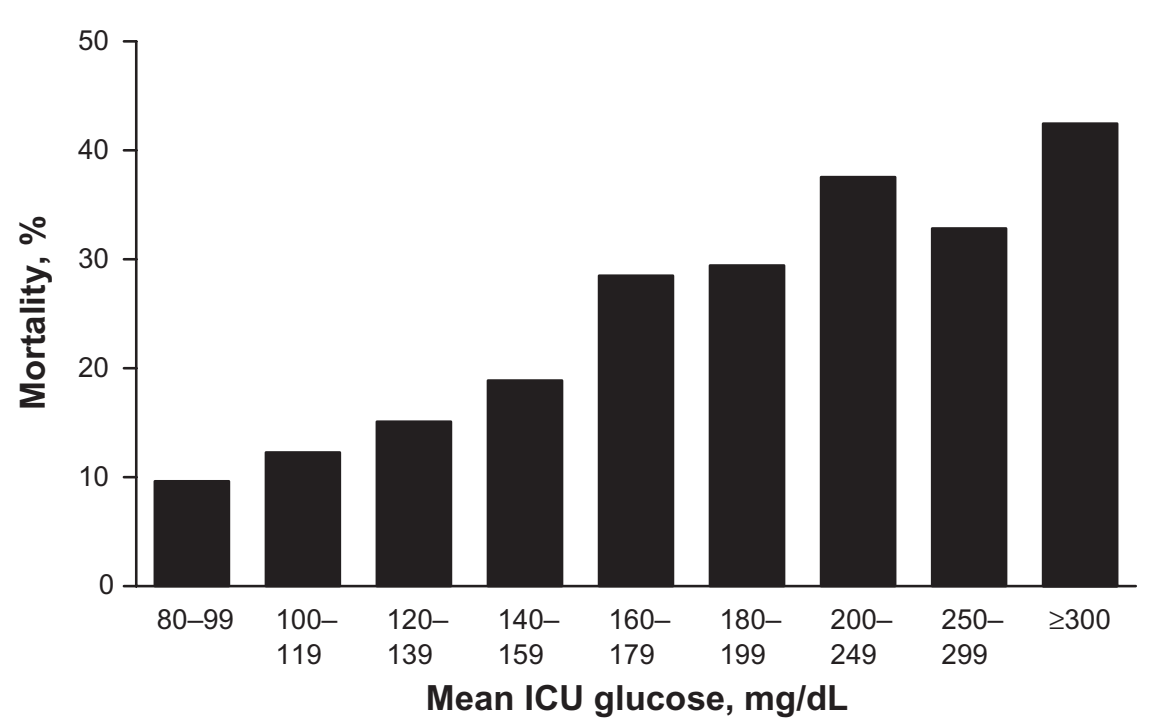

Figure I ICU mean glucose value and hospital mortality. ${ }^{21}$ Glucose values shown as a range of mean values; Chi-square test used for trend $(P<0.00 \mathrm{I})$ analysis. Abbreviation: ICU, intensive care unit.

Unfortunately, many critically ill patients are still not evaluated for hyperglycemia or unrecognized diabetes on admission to hospital, and hyperglycemia is not routinely managed aggressively. Levetan et $\mathrm{al}^{22}$ screened 1034 consecutive hospitalized adult patients without a prior history of diabetes for glucose values $>200 \mathrm{mg} / \mathrm{dL}$, and found that $37.5 \%$ of medical patients and $33 \%$ of surgical patients had hyperglycemia, although documentation of abnormal glycemia was found in just one-third of their hospital records, and only three discharge reports suggested a possible diagnosis of diabetes. Furthermore, the investigators, on following up the hospitalized patients postdischarge, found that what was perceived in the hospital as stress hyperglycemia associated with hospitalization may in fact have been frank diabetes; glucose elevations were sustained in a number of these patients over several months of follow-up after hospital discharge..$^{22,23}$

\section{Mortality, diabetes, and hyperglycemia in cardiovascular patients}

Type 2 diabetes mellitus is a well documented risk factor for cardiovascular mortality and predicts poor outcome after myocardial infarction. ${ }^{19}$ As shown in the Norfolk cohort of the European Prospective Investigation of Cancer study, even a moderately elevated $\mathrm{HbA}_{1 \mathrm{c}}$ concentration, although within the normal range, indicated an increased risk of cardiovascular disease and mortality. ${ }^{24}$ Critically ill patients with new-onset hyperglycemia, in addition, appear to have significantly higher inhospital mortality and worse functional outcomes than those with previously diagnosed diabetes or normoglycemia (Figure 2), with even modest elevations in mean glucose level contributing to increased risk for inhospital death. ${ }^{5}$

The relationship between glucose level and mortality postmyocardial infarction was first recognized in the mid 1970s. In 1988, Malmberg and Rydén ${ }^{25}$ documented a statistically significant increase in post-myocardial infarction mortality among patients with diabetes compared with those without diabetes both during the acute hospital stay $(25 \%$ versus $16 \%$; $P<0.02)$ and over the year following the event (53\% versus $28 \% ; P<0.001)$. The authors also noted a greater tendency of fatal reinfarction in the diabetic group (30\% versus $14 \%$; $P<0.05)$. These data have been substantiated and expanded upon by results from several other trials, demonstrating that hyperglycemia with or without established diabetes increased mortality risk after acute myocardial infarction or other forms of cardiovascular disease (including coronary artery disease, coronary artery bypass grafting surgery, or stroke). ${ }^{20,26-30}$ Thus, it appears that hyperglycemia, rather than diabetes status per se, influences morbidity and mortality risk.

However, results from another study of high-risk patients with diabetes and cardiovascular disease undergoing percutaneous coronary intervention found no significant difference in cardiovascular outcomes (including death) in patients with preoperative $\mathrm{HbA}_{1 \mathrm{c}}$ levels of $\leq 7.0 \%$ or $>7.0 \%{ }^{31}$ These findings may support the notion that a history of hyperglycemia imposes a long-term risk of adverse cardiovascular events even after $\mathrm{HbA}_{1 \mathrm{c}}$ is managed back to normal levels. 


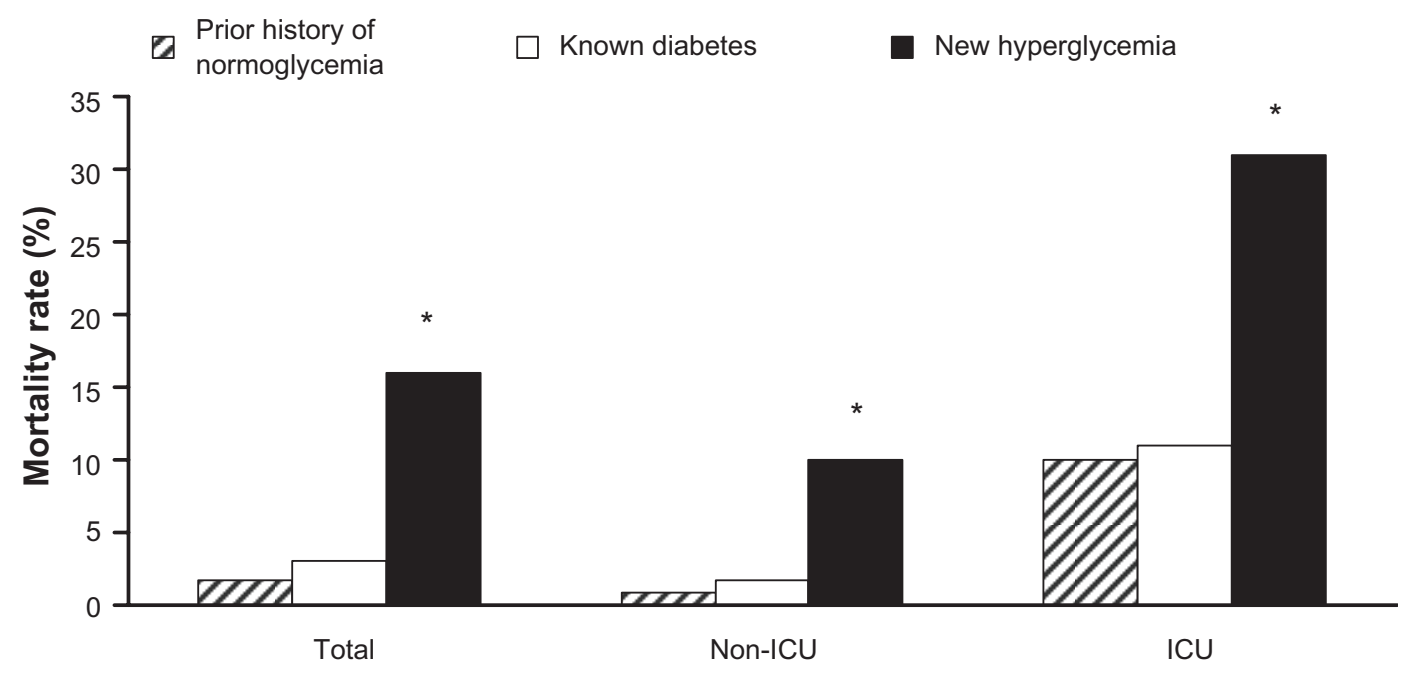

Figure 2 Effect of hyperglycemia on hospital mortality.

Note: $* P<0.01$ compared with normoglycemia and known diabetes. Copyright @ 2002, The Endocrine Society. Adapted with permission from Umpierrez GE, Isaacs SD, Bazargan N, You X, Thaler LM, Kitabchi AE. Hyperglycemia: An independent marker of in-hospital mortality in patients with undiagnosed diabetes. J Clin Endocrinol Metab. 2002;87(3):978-982. ${ }^{5}$

Abbreviation: ICU, intensive care unit.

Temporal patterns in glucose excursions may be predictive of adverse cardiac events. In one study, persistent increases in fasting glucose over the course of hospitalization for acute myocardial infarction was more predictive of increased mortality than admission fasting glucose alone, regardless of diabetic status at admission (Figure 3). ${ }^{32}$
Results from this study also showed that while outcomes improved in patients whose increased fasting glucose at admission was normalized at discharge, they remained at increased risk of long-term mortality compared with patients with persistently normal fasting glucose levels (Figure 3). ${ }^{32}$

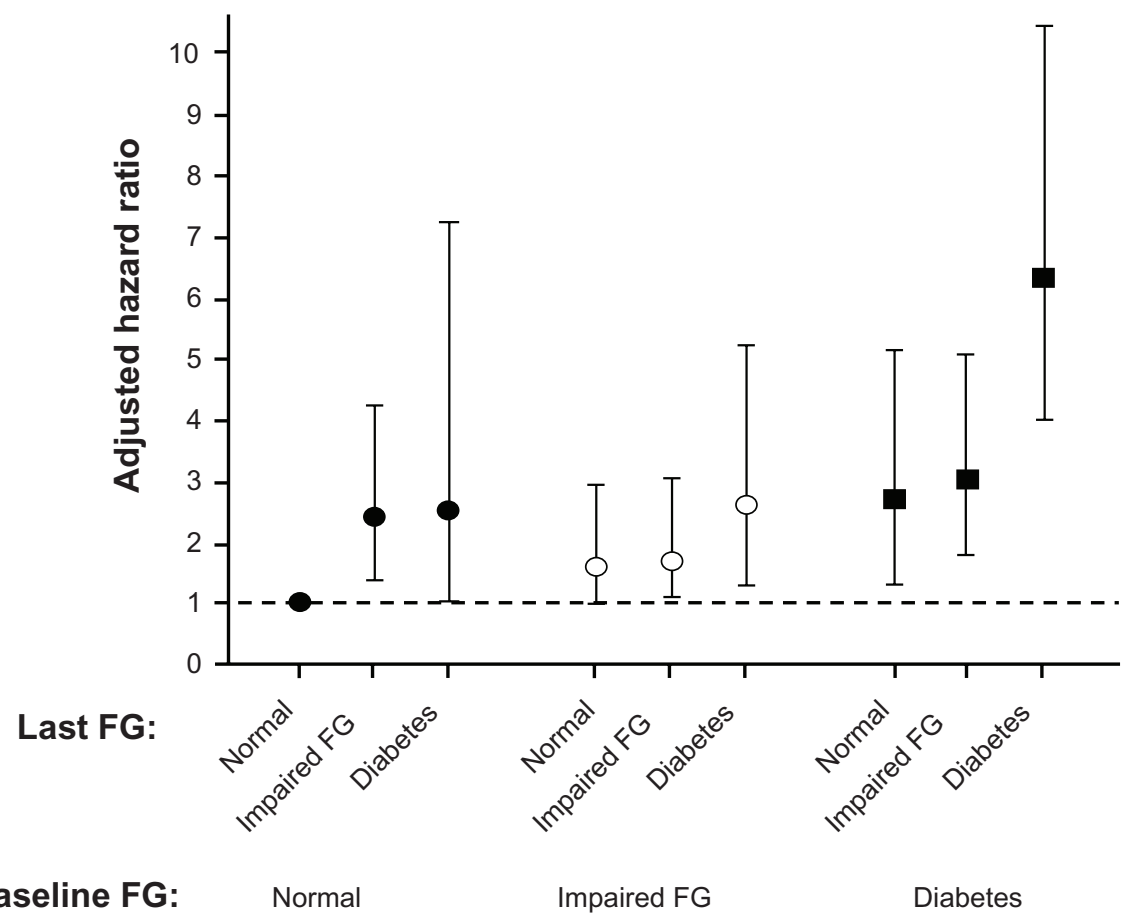

Figure 3 The effect of changes in fasting glucose levels on the Global Registry of Acute Coronary Events Score-adjusted hazard ratio for mortality. Copyright (c) 2009, The American Journal of Cardiology. Used with permission from Aronson D, Hammerman H, Suleiman M, Markiewicz W. Usefulness of changes in fasting glucose during hospitalization to predict long-term mortality in patients with acute myocardial infarction. Am J Cardiol. 2009;104(8):1013-1017.32

Abbreviation: FG, fasting glucose. 


\section{Controlling glucose in cardiovascular disease}

Patients in the Leuven studies who had undergone cardiac surgery were subsequently evaluated in a subgroup analysis, with long-term follow-up of high-risk cardiac patients. The investigators assessed outcomes in this population four years after the original admission and reported that the benefit of intensive insulin therapy in the prolonged-stay patients ( $\geq$ three days in ICU) was sustained, ie, only $23.1 \%$ of intensive treatment patients had died at four years compared with $36.2 \%$ of patients in the conventional insulin therapy group $(P=0.03)$. The authors concluded that tighter short-term glycemic control during intensive care yielded long-term survival benefits in a cardiovascular population. ${ }^{33}$

The Diabetes and Insulin-Glucose Infusion in Acute Myocardial Infarction (DIGAMI) study established that survival rates among post-myocardial infarction patients with diabetes could be improved if they were treated to normoglycemia with insulin therapy. ${ }^{34}$ In this study, 620 patients who had experienced a myocardial infarction in the previous 24 hours and had a blood glucose level $>198.2 \mathrm{mg} / \mathrm{dL}$ (with or without documented diabetes) received either an insulinglucose infusion followed by subcutaneous insulin dosing four times daily for three months or longer or conventional insulin therapy (standard care, with insulin given when indicated). At one year, patients who received aggressive treatment experienced an approximately $30 \%$ relative reduction in mortality compared with conventional insulin therapy $(P=0.027)$. The advantage was even greater among subjects with a low cardiovascular risk profile and no prior insulin therapy ( $52 \%$ reduction in mortality; $P=0.046) .{ }^{34}$ This benefit was sustained at 3.5 years' follow-up, when an absolute reduction in mortality of $11 \%$ was observed. ${ }^{35} \mathrm{~A}$ follow-up trial, DIGAMI 2, failed to replicate these results, ${ }^{36}$ likely because the intensive treatment group did not achieve significantly improved glucose control compared with conventional insulin therapy. ${ }^{37}$ However, DIGAMI 2 did show the admission blood glucose level to be a strong independent predictor of mortality following acute myocardial infarction. ${ }^{36}$

Furnary et al ${ }^{29}$ evaluated the benefit of tight glycemic control among patients with diabetes undergoing coronary artery bypass grafting. Continuous insulin infusion via the Portland Protocol was used to maintain blood glucose levels between 100 and $150 \mathrm{mg} / \mathrm{dL}$ in the perioperative period; this was compared with historical controls given subcutaneous insulin treatment to sustain glucose levels $<200 \mathrm{mg} / \mathrm{dL}$. Of 3554 patients studied, mortality was significantly lower in those treated with continuous insulin infusion $(P<0.0001)$. Recent work from this group suggested that three postoperative days of excellent glycemic control (blood glucose $<150 \mathrm{mg} / \mathrm{dL}$ ) decreased the risk of deep sternal wound infection by $77 \%$, mortality by $60 \%$, and length of stay, thus apparently eliminating the incrementally increased risk of complications previously seen in patients with diabetes. ${ }^{38}$

Recently, results of the Action to Control Cardiovascular Risk in Diabetes (ACCORD), ${ }^{39}$ the Veterans Affairs Diabetes Trial (VADT) ${ }^{40}$ and Action in Diabetes and Vascular Disease: Preterax and Diamicron MR Controlled Evaluation (ADVANCE) $)^{41}$ trials have been published, indicating that intensive glycemic control (to target $\mathrm{HbA}_{1 \mathrm{c}}<6.0 \%$ or $\leq 6.5 \%$, respectively) in patients with type 2 diabetes mellitus at high cardiovascular risk failed to reduce major cardiovascular events and, in fact, was associated with higher mortality in the ACCORD study. The exact reason for the increase in mortality is not clear, but a similar median $\mathrm{HbA}_{1 \mathrm{c}}$ concentration was achieved in the ADVANCE study with no increased mortality. ${ }^{42}$ An additional analysis of the ACCORD data suggests that an $\mathrm{HbA}_{1 \mathrm{c}}$ concentration of $<7.0 \%$ alone cannot account for the increased mortality risk nor is it a predictor of mortality risk. ${ }^{43}$ While these studies leave many questions unanswered, it is important to note that patients in each study arm were treated with a variety of glucose-lowering drugs rather than defined regimens; thus, these studies were not specifically testing the effects of insulin therapy. In fact, only $40 \%$ of subjects in the intensive control arm of ADVANCE were prescribed insulin. ${ }^{41}$ In their appraisal of these three trials, the American Diabetes Association and the American Heart Association did not suggest major changes in glycemic control targets but rather emphasized individualized care. ${ }^{42} \mathrm{~A}$ recent retrospective cohort analysis of almost 28,000 patients with type 2 diabetes mellitus from the UK General Practice Research Database showed the lowest and highest achieved $\mathrm{HbA}_{1 \mathrm{c}}$ concentrations (median $\mathrm{HbA}_{1 \mathrm{c}} 6.4 \%$ and $10.5 \%$, respectively) were associated with an increased risk of all-cause mortality for patients whether they were treated with oral therapy or insulin $(1.52,95 \%$ confidence interval [CI]: 1.32-1.76], $P<0.0001 ; 1.79$ [95\% CI: 1.56-2.06], $P<0.0001$, respectively). ${ }^{44}$ The authors posit that this increase in mortality in the lowest $\mathrm{HbA}_{1 \mathrm{c}}$ group might have been associated with hypoglycemia because mortality was higher in patients who had severe hypoglycemia compared with those without severe hypoglycemia. These study results also support recommendations for an $\mathrm{HbA}_{1 \mathrm{c}}$ target value that differs depending on the individual patient. ${ }^{45}$ 
One ongoing trial is evaluating the impact of insulin therapy on cardiovascular outcomes in patients with cardiovascular disease who are not hospitalized. In the Outcome Reduction with Initial Glargine Intervention (ORIGIN) trial, investigators aim to determine whether maintaining normoglycemia (fasting glucose $<95 \mathrm{mg} / \mathrm{dL}$ ) with insulin glargine can reduce the risk of cardiovascular morbidity and/or mortality among outpatients with impaired fasting glucose, impaired glucose tolerance, or early type 2 diabetes mellitus who are at high risk for vascular disease. ${ }^{46}$ The goal of the Impact of Tight Glycemic Control in Acute Myocardial Infarction trial is to assess whether aggressive insulin therapy can improve myocardial function and perfusion and alter vascular risk factors in patients with acute myocardial infarction and hyperglycemia. ${ }^{47}$

\section{Controlling glucose in critical and intensive care patients}

Several landmark studies have shown that insulin therapy to achieve tight glucose control improves acute and long-term outcomes in critically ill adults. In the first Leuven study, van den Berghe et $\mathrm{al}^{8}$ studied 1548 surgical ICU patients randomized to intensive insulin therapy (to achieve blood glucose levels of $80-110 \mathrm{mg} / \mathrm{dL}$ ) or conventional therapy (insulin at blood glucose levels $>215 \mathrm{mg} / \mathrm{dL}$ to reach $180-200 \mathrm{mg} / \mathrm{dL}$ ). At 12 months, a $42 \%$ reduction in ICU mortality was observed, and overall inhospital mortality decreased by $34 \%$ with intensive insulin therapy $(P<0.01)$. The benefit was especially apparent in patients who remained in the ICU for more than five days (10.6\% versus $20.2 \%$ with intensive versus conventional treatment, respectively; $P=0.005)$. Intensive therapy also reduced morbidity, including the number of episodes of septicemia, acute renal failure, red blood cell transfusions, and critical illness neuropathy (Figure 4). ${ }^{8}$

A later Leuven study of 1200 adult medical ICU patients (767 of whom stayed in the ICU for at least a third day) followed the same protocol for intensive versus conventional insulin treatment. ${ }^{13}$ Morbidity was significantly reduced among patients on intensive therapy $(P=0.04)$ and, although overall mortality did not differ between groups, inhospital mortality was significantly reduced among patients under ICU care for three days or more $(52.5 \%$ versus $43 \%$ for conventional versus intensive insulin therapy, respectively; $P=0.009) .{ }^{13}$ However, results also indicated more frequent hypoglycemia in the intensive treatment group $(3.1 \%$ versus $18.7 \%$ for conventional versus intensive insulin therapy; $P<0.001)$. Thus, benefits of tight glycemic control were again shown to be greatest among patients who had more severe illness and required a longer ICU stay. Increased risk of hypoglycemia may be an expected consequence of such studies, although there was no evidence of clinically

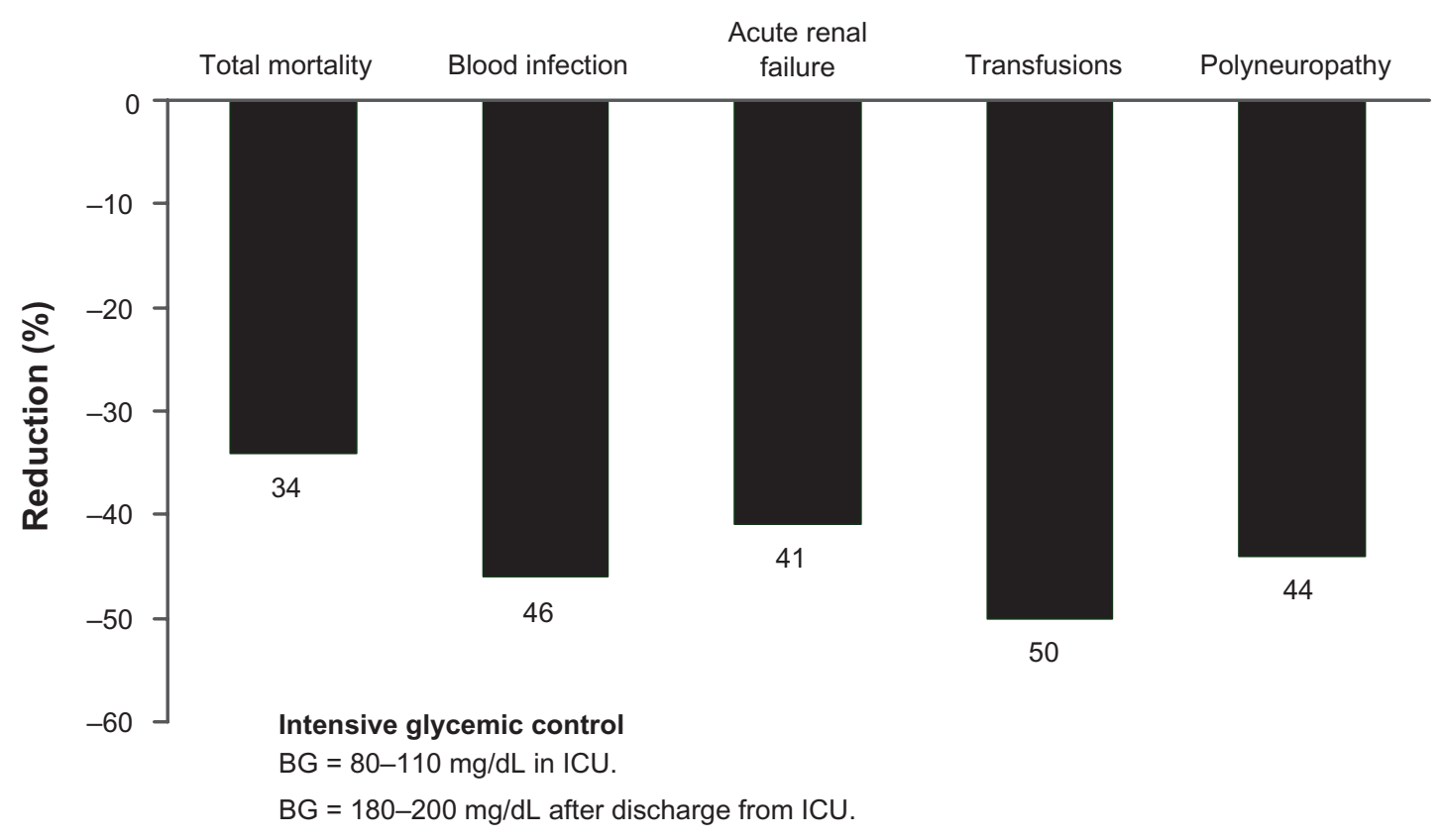

Figure 4 Intensive insulin infusion in critically ill hospitalized patients: reduced morbidity and mortality. ${ }^{8}$ Abbreviations: BG, blood glucose; ICU, intensive care unit. 
significant harm as a result. However, two intensive insulin therapy trials, ie, the European Glucontrol trial and the Volume Substitution and Insulin Therapy in Severe Sepsis (VISEP) trial, were halted because of such safety concerns. The Glucontrol trial evaluated the role of intensive insulin therapy $(4.4-6.1 \mathrm{mmol} / \mathrm{L})$ or "intermediate glucose control" insulin therapy $(7.8-10.0 \mathrm{mmol} / \mathrm{L})$ in medical ICU patients. ${ }^{48}$ The trial was halted because of a near fourfold increase in hypoglycemia associated with intensive insulin therapy, with no significant survival benefit compared with intermediate glucose control. In the VISEP trial, ICU patients with severe sepsis were randomized to intensive or conventional insulin therapy. ${ }^{49}$ At 28 days, there was a statistically significant, nearly fourfold increase in the rate of severe hypoglycemia (defined as $2.2 \mathrm{mmol} / \mathrm{L}$ [ $\leq 40 \mathrm{mg} / \mathrm{dL}]$ ) that was considered to be life-threatening. ${ }^{49,50} \mathrm{In}$ another study, Arabi et al ${ }^{51}$ found that there was no statistically significant increase in mortality in ICU patients with hypoglycemia except in patients with severe hypoglycemia $(\leq 1.2 \mathrm{mmol} / \mathrm{L})$ and in patients with an admission blood glucose level of $\leq 10 \mathrm{mmol} / \mathrm{L}$.

The large Normoglycemia in Intensive Care EvaluationSurvival Using Glucose Algorithm Regulation (NICESUGAR) trial treated patients $(\mathrm{n}=6104)$ who were expected to be in the ICU for at least three days with either intensive glycemic control (defined as a blood glucose target of $81-108 \mathrm{mg} / \mathrm{dL}$ ) or conventional glycemic control (target of $144-180 \mathrm{mg} / \mathrm{dL}$ ) using intravenous insulin. ${ }^{12}$ There was a significant difference between the intensive and conventional treatment arms for death at 90 days $(27.5 \%$ versus $24.9 \%$; odds ratio: 1.14 [95\% CI: $1.02-1.28$ ], respectively; $P=0.02$ ), and a significant increase in severe hypoglycemia $(<40 \mathrm{mg}$ / dL) was reported in the intensive group compared with the conventional group $(6.8 \%$ versus $0.5 \% ; P<0.001)$. The lack of benefits in the NICE-SUGAR study may not be because of reduced glucose levels, which were modest (145 versus $118 \mathrm{mg} / \mathrm{dL}$ on average), but may stem from the use of insulin with a high relative rate of hypoglycemia and other factors specific to the NICE-SUGAR treatment regimens. A large meta-analysis of 26 trials involving over 13,567 patients, and which included the NICE-SUGAR data, was conducted to clarify the influence of intensive insulin therapy compared with conventional insulin therapy on mortality and severe hypoglycemia in ICU. ${ }^{14}$ Results showed that intensive insulin therapy in the ICU setting had no effect on overall risk of 90-day mortality. There was a sixfold increased risk of severe hypoglycemia among patients given intensive insulin therapy compared with the control treatment regardless of the type of ICU or the intensity of insulin therapy (Figure 5). , $12-14,49,52-73^{-1}$
However, further analysis revealed significant heterogeneity among the included trials, based on type of ICU unit and target glucose levels. When these variables were included in the analysis model, it was found that surgical ICU patients (versus medical ICU) given intensive insulin therapy showed a significantly lower relative risk of 90 -day mortality (relative risk: $0.63,95 \% \mathrm{CI}$ : $0.44-0.91 ; P=0.02$ ). This meta-analysis highlights the inconsistent outcomes observed in critically ill patients given intensive insulin therapy. The variability in the literature is likely due to key methodologic differences among the available trials, including widely different criteria for "usual" glycemic control (>10 mmol/L to $6.0-8.0 \mathrm{mmol} / \mathrm{L})$ versus "intensive" control $(4.0-8.3 \mathrm{mmol} / \mathrm{L}) .{ }^{14}$ Further research is required to define optimal glycemic targets and insulin regimens better, and appropriate selection criteria for managing hyperglycemia in subgroups of ICU patients.

Intensive insulin therapy in special populations has been shown to improve key clinical outcomes. A study of 700 critically ill patients admitted to the pediatric ICU found that intensive insulin therapy resulted in a statistically significant reduction in blood glucose levels, duration of pediatric ICU stay, and the inflammatory marker, C-reactive protein, but significantly more hypoglycemic events compared with conventional insulin therapy. There were significantly fewer deaths in the intensive insulin therapy group and, after correction for baseline factors, hypoglycemia (defined as either blood glucose $\leq 2.2 \mathrm{mmol} / \mathrm{L}$ or $<1.7 \mathrm{mmol} / \mathrm{L}$ [ $\leq 39.6 \mathrm{mg} / \mathrm{dL}$ or $<30.6 \mathrm{mg} / \mathrm{dL}$ ]) was not an independent predictor of mortality. ${ }^{74}$

Patients with traumatic brain injuries or acute stroke are at increased risk of stress-related hyperglycemia. In a study of 240 patients with traumatic brain injury, intensive insulin therapy reduced infection rates, shortened the patient's stay in ICU and improved neurologic outcomes compared with conventional insulin therapy. Hypoglycemic events occurred in only seven patients. However, there was no significant difference in mortality between the two groups at six months follow-up. ${ }^{75}$ With acute stroke, approximately $40 \%$ of patients will exhibit hyperglycemia for several days following the event. ${ }^{76}$ In a small sample of acute ischemic stroke patients, higher mean blood glucose levels monitored continuously over 72 hours post-stroke $(7 \mathrm{mmol} / \mathrm{L})$ were correlated with larger infarct size and poorer functional outcomes. ${ }^{77}$ In one meta-analysis ( $\mathrm{n}=32$ trials), short-term mortality was threefold higher, and poorer functional outcome was seen among nondiabetic patients with moderately elevated glucose levels $(>6.1 \mathrm{mmol} / \mathrm{L})$ following ischemic stroke. ${ }^{78}$ To date, there is no clear evidence of a clinical benefit of 


\begin{tabular}{|c|c|c|c|c|c|}
\hline \multicolumn{4}{|c|}{ No. Deaths/Total No. Patients } & \multirow{2}{*}{ Favors IIT } & \multirow{2}{*}{$\stackrel{\text { Favors control }}{\longrightarrow}$} \\
\hline Study & IIT & Control & Risk ratio $(95 \% \mathrm{Cl})$ & & \\
\hline \multicolumn{6}{|l|}{ Mixed ICU } \\
\hline Yu et al52 & $4 / 28$ & $4 / 27$ & $0.96(0.27-3.47)$ & & \\
\hline Henderson et $a^{53}$ & $5 / 32$ & $7 / 35$ & $0.78(0.28-2.22)$ & & \\
\hline Mitchell et al ${ }^{54}$ & $9 / 35$ & $3 / 35$ & $3.00(0.89-10.16)$ & & \\
\hline Wang et $a^{55}$ & $7 / 58$ & $26 / 58$ & $0.27(0.13-0.57)$ & & \\
\hline Azevedo et $\mathrm{al}^{56}$ & $38 / 168$ & $42 / 169$ & $0.91(0.62-1.34)$ & & \\
\hline McMullin et al ${ }^{57}$ & $6 / 11$ & $4 / 9$ & $1.23(0.49-3.04)$ & & \\
\hline Devos et al ${ }^{58}$ & $107 / 550$ & $89 / 551$ & $1.20(0.93-1.55)$ & & \\
\hline Brunkhorst et al ${ }^{49}$ & $98 / 247$ & $102 / 288$ & $1.12(0.90-1.39)$ & & \\
\hline lapichino et al ${ }^{59}$ & $15 / 45$ & $12 / 45$ & $1.25(0.66-2.36)$ & & \\
\hline He et $a^{60}$ & $16 / 58$ & $29 / 64$ & $0.61(0.37-1.00)$ & & \\
\hline Zhang et al ${ }^{61}$ & $4 / 168$ & $6 / 170$ & $0.67(0.19-2.35)$ & & \\
\hline De La Rosa Gdel et al ${ }^{62}$ & $102 / 254$ & $96 / 250$ & $1.05(0.84-1.30)$ & & \\
\hline Arabi et $a^{63}$ & $72 / 266$ & $83 / 257$ & $0.84(0.64-1.09)$ & & \\
\hline Mackenzie et $a^{64}$ & $39 / 121$ & $47 / 119$ & $0.82(0.58-1.15)$ & & \\
\hline NICE-SUGAR ${ }^{12}$ & $829 / 3010$ & $751 / 3012$ & $1.10(1.01-1.20)$ & & \\
\hline All mixed ICU patients & $1351 / 5051$ & $1301 / 5089$ & $0.99(0.87-1.12)$ & & \\
\hline \multicolumn{6}{|l|}{ Medical ICU } \\
\hline Bland et al ${ }^{65}$ & $1 / 5$ & $2 / 5$ & $0.50(0.06-3.91)$ & & \\
\hline Van den Berghe et al ${ }^{13}$ & $214 / 595$ & $228 / 605$ & $0.95(0.82-1.11)$ & & \\
\hline Walters et al ${ }^{66}$ & $1 / 13$ & $0 / 12$ & $2.79(0.12-62.48)$ & & \\
\hline Farah et al ${ }^{67}$ & $22 / 41$ & $22 / 48$ & $1.17(0.77-1.78)$ & & \\
\hline Oksanen et al ${ }^{68}$ & $13 / 39$ & $18 / 51$ & $0.94(0.53-1.68)$ & & \\
\hline Bruno et al ${ }^{69}$ & $2 / 31$ & $0 / 15$ & $2.50(0.13-49.05)$ & & \\
\hline \multirow{2}{*}{\multicolumn{2}{|c|}{ Surgical ICU }} & $270 / 736$ & $1.00(0.78-1.28)$ & & \\
\hline & $55 / 765$ & $85 / 783$ & $0.66(0.48-0.92)$ & & \\
\hline Grey et al70 & $4 / 34$ & $6 / 27$ & $0.53(0.17-1.69)$ & & \\
\hline Bilotta et $\mathrm{al}^{71}$ & $6 / 40$ & $7 / 38$ & $0.81(0.30-2.20)$ & & \\
\hline He et $\mathrm{al}^{72}$ & $7 / 150$ & $6 / 38$ & $0.30(0.11-0.83)$ & & \\
\hline Bilotta et $\mathrm{al}^{73}$ & $5 / 48$ & $6 / 49$ & $0.85(0.28-2.60)$ & & \\
\hline All surgical ICU patients & $77 / 1037$ & $110 / 935$ & $0.63(0.44-0.91)$ & & \\
\hline \multirow[t]{2}{*}{ All ICU patients } & $1681 / 6812$ & $1681 / 6760$ & $0.93(0.83-1.04)$ & & \\
\hline & & & & & 1 \\
\hline
\end{tabular}

Figure 5 Effect of intensive insulin therapy versus conventional insulin therapy on mortality in intensive care unit patients. Tests for heterogeneity: mixed ICU: $Q$ statistic, 29.54 ( $P<0.01$ ), 12, 52.6\%; medical ICU: Q statistic, 2.05 ( $P=0.84), 12,0.0 \%$; surgical ICU: Q statistic, $2.78(P=0.60)$, 12 statistic, $0.0 \%$; all ICU patients: Q statistic, 46.67 $(P<0.01), 12,46.4 \%$. Copyright $(2) 2009$, Access Copyright Used with permission from Griesdale DE, de Souza RJ, van Dam RM, et al. Intensive insulin therapy and mortality among critically ill patients: A meta-analysis including NICE-SUGAR study data. CMAJ. 2009; I80(8):82I-827.14

Abbreviations: Cl, confidence interval; NICE-SUGAR, Normoglycemia in Intensive Care Evaluation-Survival Using Glucose Algorithm Regulation; ITT, intensive insulin therapy.

insulin therapy during acute stroke treatment. In the largest trial to date (Glucose Insulin in Stroke Trial, $\mathrm{n}=933$ ), stroke patients admitted with hyperglycemia and randomized to receive 24 hours of continuous glucose potassium and insulin showed an all-cause mortality rate and functional outcomes similar to patients who were randomized to receive placebo. ${ }^{79}$

There are many variables that factor into the risks and benefits of intensive insulin therapy in the hospital setting. These include differences in patient population, disease severity, different protocols, different glycemic targets, different definitions of hypoglycemia, and different underlying comorbidities. While each of these can directly impact the results of a randomized clinical trial, their clinical significance remains to be fully elucidated. Another possibly important factor that may impact patient outcome with insulin therapy is blood glucose variability, which has gained attention in recent years after observations of heightened risk of hypoglycemia with intensive insulin therapy. The evidence indicates that wide fluctuation in blood glucose concentrations increases risk of mortality and other poor outcomes in critically ill patients. ${ }^{80-84}$ In one of the earliest of these studies, ${ }^{81}$ researchers derived standard deviation values as a marker of glucose variability from large databases of blood glucose measurements in critically ill patients $(n=7049)$. Analysis showed significantly higher blood glucose standard deviation values among nonsurvivors compared with survivors. Logistic regression revealed a positive and independent predictive relationship between glucose variability and risk of mortality that was stronger than that observed between mortality and mean glucose concentration. ${ }^{81}$ More recently, Dossett et $\mathrm{al}^{83}$ and Al-Dorzi et $\mathrm{al}^{84}$ observed similar predictive relationships between glucose variability and mortality in surgical ICU patients. In the study from Al-Dorzi et al, ${ }^{84}$ daytime glucose fluctuation (the difference between the highest and lowest glucose values) was similar among patients $(n=523)$ who were randomized to either intensive or conventional insulin therapy. Regardless of 
insulin therapy group, those patients with wide glycemic fluctuation (versus narrow fluctuation) exhibited a higher likelihood of mortality (22.2\% versus $8.4 \% ; P<0.001)$, even when controlling for patient age, diabetic status, and daily insulin dose ${ }^{84}$ Further research is needed to determine whether reducing glycemic fluctuation improves patient outcomes.

\section{Guidelines for insulin therapy in critically ill hospitalized patients}

The American Diabetes Association has issued guidelines for managing diabetes in critically ill patients in the hospital setting. ${ }^{15}$ The recommendations for blood glucose levels for critically ill patients target an initial glucose level of $\leq 180 \mathrm{mg} / \mathrm{dL}(10.0 \mathrm{mmol} / \mathrm{L})$, with intravenous infusion of insulin being the preferred route of administration. ${ }^{15}$ After initiation of intravenous insulin, glucose level should be maintained between 140 and $180 \mathrm{mg} / \mathrm{dL}$ (7.8 and $10.0 \mathrm{mmol} / \mathrm{L})$. Glycemic targets of $<110 \mathrm{mg} / \mathrm{dL}$ $(6.1 \mathrm{mmol} / \mathrm{L})$ are not recommended. ${ }^{15}$ Complete descriptions of recommended insulin infusion methods are available in American Diabetes Association guidelines and other sources. ${ }^{37,85-89}$

Successful protocols for intravenous insulin administration all share a number of features, including established efficacy with minimal hypoglycemia risk, adaptability to all hospital units, and ease of implementation. Algorithms designed and standardized to the needs of a multidisciplinary team, including physicians, nurses, pharmacists, and cardiologists, and implemented by nursing staff, are becoming more common, because they ensure consistency of care with cost-conscious appeal..$^{85,87,90-95}$ In addition, strategies for addressing hypoglycemia episodes should be a standard component of infusion protocols, preferably set up in advance and implemented by a well-trained multidisciplinary team. Many hypoglycemia episodes can be forestalled by coordinating nutrition (eg, tube feeding, total parenteral nutrition) and insulin therapy. ${ }^{85,86}$

As the condition of critically ill patients improves, they are transitioned into the general hospital population. Observational studies suggest that, like critically ill patients, hyperglycemia in the general hospital inpatient is associated with poorer outcomes, including increased mortality (versus normoglycemic patients). ${ }^{5}$ There are currently no prospective investigations of illness or functional outcomes, however, with glycemic management in noncritically ill inpatients with hyperglycemia. Moreover, in the general hospital setting, highly skilled nursing staff specially trained to implement intravenous insulin therapy regimens and invasive monitoring techniques are not available. Nevertheless, based on the available data, the American Diabetes Association recommends the active management of hyperglycemia in the non-ICU setting. The joint American Diabetes Association and American Association of Clinical Endocrinologists guidelines recommend glycemic management in ICU patients transitioning to general inpatient care, as well as noncritically ill patients who exhibit premeal blood glucose levels of $>140 \mathrm{mg} / \mathrm{dL}(7.8 \mathrm{mmol} / \mathrm{L}) .{ }^{15}$ For most inpatients, routine bedside blood glucose monitoring performed prior to meals and at bedtime can identify patients with hyperglycemia. ${ }^{4}$ In the general inpatient setting, scheduled subcutaneous insulin is the preferred method for achieving desired glycemic targets. Such a regimen should include basal, nutritional, and supplemental (eg, corrective) components. ${ }^{15}$ Successful implementation of a subcutaneous insulin regimen to achieve recommended glycemic targets safely in the non-ICU general hospital setting is possible given proper staff training and systems coordination so that prandial insulin doses are adjusted appropriately in accord with meal delivery timing, content, and patient consumption. ${ }^{4}$ Appropriately selected ICU patients will need to transition to subcutaneous insulin administration. ${ }^{4,15}$ Although there are no data identifying the best protocol for transitioning to subcutaneous insulin, the American Diabetes Association and American Association of Clinical Endocrinologists suggest that $75 \%-80 \%$ of the total daily intravenous infusion dose be divided into basal and prandial components. The use of basal insulin is widely recommended to expedite the transition to subcutaneous insulin therapy from intravenous insulin therapy and to maintain glucose control; ${ }^{4}$ a long-acting analog, such as insulin glargine or insulin detemir, may be the best option. ${ }^{86}$ The long-acting formulations can address basal needs while minimizing the risk of hypoglycemia, which is a significant challenge with shorter-acting analogs. ${ }^{96,97}$ To prevent hyperglycemia, subcutaneous insulin must be initiated $1-4$ hours prior to discontinuation of the intravenous insulin. ${ }^{15}$

\section{Effective hospital discharge}

Transitioning care from the inpatient to the outpatient setting is important to achieve better glycemic control and improve outcomes. ${ }^{98}$ For the patient with newly diagnosed diabetes, effective discharge includes providing the necessary patient education and skills training and a clear postdischarge plan that is well documented and easily accessible to the patient's outpatient clinicians. ${ }^{98}$ In addition, patients should have a basic understanding of the disease and insulin administration, 
be able to perform glucose self-monitoring, and be aware of the risks of hypoglycemia. ${ }^{99}$ These individuals should receive clear written instructions, which will be a useful at-home guide and reference for both outpatients and their caregivers. More comprehensive diabetes self-care education should be arranged in the community outpatient setting. It is also critical for hospital staff to communicate with outpatient care providers, either directly or via a hospital discharge summary, particularly with regard to a care plan, complications, and comorbidities. ${ }^{15}$ Patients with diabetes should be instructed to see their primary care clinician within seven to 30 days of discharge. For normoglycemic individuals who experienced stress hyperglycemia during their illness, it will be critical to determine whether hyperglycemia resolves and remains at normal levels postdischarge. These patients should see their clinicians one month postdischarge. ${ }^{99}$ While no formal recommendations have been published regarding postdischarge follow-up for such patients, it seems reasonable to suggest that they self-monitor blood glucose levels at home in the interim period between discharge and follow-up with their physician. If the patient detects hyperglycemia during this period, they should contact their care provider.

\section{Summary}

Hyperglycemia and large variability in glycemic indices increases the risk of morbidity and mortality substantially among critically ill patients and those with cardiovascular disease. A significant body of evidence supports the use of insulin therapy for glucose control in appropriately selected critically ill patients. It is now recommended that all patients receive glucose testing on admission to the hospital and glucose-lowering therapy if hyperglycemia is found. Many organizations, including the American Diabetes Association and the American Association of Clinical Endocrinologists, endorse glycemic control for hospitalized patients and support the use of proven insulin infusion protocols and hypoglycemia prevention measures to achieve normoglycemia safely in this population. While conventional glucose-lowering interventions that aim to maintain blood glucose levels between $140-180 \mathrm{mg} / \mathrm{dL}$ are recommended for critically ill patients, ${ }^{4}$ intensive glucose-lowering protocols having target glucose levels set at $<110 \mathrm{mg} / \mathrm{dL}$ remain controversial. Benefits with such protocols, in terms of increased survival and shorter ICU stays, have been shown in surgical ICU patients ${ }^{14}$ and those who remained in the ICU for three days or longer, ${ }^{13}$ benefits have not been observed in other patient groups and more frequent hypoglycemia has been reported. Given these inconsistent findings, further investigation of aggressive glucose-lowering therapy in specific patient populations is required.

Inpatient management is only the first phase in a new paradigm of glycemic control. Persistent hyperglycemia or postdischarge diabetes must be identified to allow for blood glucose control after hospital discharge; this should be an integral part of the comprehensive treatment plan for cardiovascular patients. Finally, providing patients with the tools and knowledge to maintain long-term normoglycemia after discharge via a self-management education program based on a safe and effective combination of insulin analog and oral antidiabetic drugs can improve the prognosis for those at continued cardiovascular risk due to uncontrolled hyperglycemia.

\section{Disclosure}

The contents of the paper and opinions expressed within are those of the author, and it was the decision of the author to submit the manuscript for publication. The author contributed to the writing of this manuscript, including critical review and editing of each draft, and approval of the submitted version. Editorial support was provided by the staff at Embryon and was funded by sanofi-aventis U.S. The author reports no conflicts of interest in this work.

\section{References}

1. Cydulka RK, Maloney GE. Diabetes mellitus and disorders of glucose homeostasis. In: Rosen's Emergency Medicine. 7th ed. St Louis, MO: Mosby Elsevier; 2009;124:1633-1649.

2. National Diabetes Information Clearinghouse, a service of the National Institute of Diabetes and Digestive and Kidney Diseases, National Institutes of Health. National Diabetes Statistics, 2007. Available from: http://diabetes.niddk.nih.gov/dm/pubs/statistics/. Accessed 2010 Sep 20

3. Saydah SH, Fradkin J, Cowie CC. Poor control of risk factors for vascular disease among adults with previously diagnosed diabetes. JAMA. 2004;291(3):335-342.

4. Moghissi ES, Korytkowski MT, DiNardo M, et al. American Association of Clinical Endocrinologists and American Diabetes Association consensus statement on inpatient glycemic control. Endocr Pract. 2009;15(4):353-369.

5. Umpierrez GE, Isaacs SD, Bazargan N, You X, Thaler LM, Kitabchi AE. Hyperglycemia: An independent marker of in-hospital mortality in patients with undiagnosed diabetes. J Clin Endocrinol Metab. 2002;87(3):978-982.

6. ACE/ADA Task Force on Inpatient Diabetes. American College of Endocrinology and American Diabetes Association consensus statement on inpatient diabetes and glycemic control: A call to action. Diabetes Care. 2006;29(8):1955-1962.

7. Clement S, Braithwaite SS, Magee MF, et al. Management of diabetes and hyperglycemia in hospitals. Diabetes Care. 2004;27(2): 553-591.

8. van den Berghe G, Wouters P, Weekers F, et al. Intensive insulin therapy in critically ill patients. N Engl J Med. 2001;345(19):1359-1367.

9. Garber AJ, Moghissi ES, Bransome ED Jr, et al. American College of Endocrinology position statement on inpatient diabetes and metabolic control. Endocr Pract. 2004;10(1):77-82. 
10. Rhoads GG, Kosiborod M, Nesto RW, et al. Comparison of incidence of acute myocardial infarction in patients with type 2 diabetes mellitus following initiation of neutral protamine Hagedorn insulin versus insulin glargine. Am J Cardiol. 2009;104(7):910-916.

11. Juhaeri J, Gao S, Dai WS. Incidence rates of heart failure, stroke, and acute myocardial infarction among Type 2 diabetic patients using insulin glargine and other insulin. Pharmacoepidemiol Drug Saf. 2009;18(6):497-503.

12. NICE-SUGAR Study Investigators, Finfer S, Chittock DR, et al. Intensive versus conventional glucose control in critically ill patients. $N \mathrm{Engl}$ J Med. 2009;360(13):1283-1297.

13. van den Berghe $G$, Wilmer A, Hermans G, et al. Intensive insulin therapy in the medical ICU. N Engl J Med. 2006;354(5):449-461.

14. Griesdale DE, de Souza RJ, van Dam RM, et al. Intensive insulin therapy and mortality among critically ill patients: A meta-analysis including NICE-SUGAR study data. CMAJ. 2009;180(8):821-827.

15. American Diabetes Association. Standards of Medical Care in Diabetes - 2010. Diabetes Care. 2010;33 Suppl 1:S11-S61

16. Shehadeh N, On A, Kessel I, et al. Stress hyperglycemia and the risk for the development of type 1 diabetes. J Pediatr Endocrinol Metab. 1997;10(3):283-286.

17. Weiss SL, Alexander J, Agus MS. Extreme stress hyperglycemia during acute illness in a pediatric emergency department. Pediatr Emerg Care. 2010;26(9):626-632.

18. Cely CM, Arora P, Quartin AA, Kett DH, Schein RM. Relationship of baseline glucose homeostasis to hyperglycemia during medical critical illness. Chest. 2004;126(3):879-887.

19. Bartnik M, Ryden L, Ferrari R, et al. The prevalence of abnormal glucose regulation in patients with coronary artery disease across Europe. The Euro Heart Survey on diabetes and the heart. Eur Heart J. 2004;25(21):1880-1890.

20. Norhammar AM, Ryden L, Malmberg K. Admission plasma glucose: Independent risk factor for long-term prognosis after myocardial infarction even in nondiabetic patients. Diabetes Care. 1999;22(11) 1827-1831.

21. Krinsley JS. Association between hyperglycemia and increased hospital mortality in a heterogeneous population of critically ill patients. Mayo Clin Proc. 2003;78(12):1471-1478.

22. Levetan CS, Passaro M, Jablonski K, Kass M, Ratner RE. Unrecognized diabetes among hospitalized patients. Diabetes Care. 1998;21(2): 246-249.

23. Norhammar A, Tenerz A, Nilsson G, et al. Glucose metabolism in patients with acute myocardial infarction and no previous diagnosis of diabetes mellitus: A prospective study. Lancet. 2002;359(9324): 2140-2144.

24. Khaw K-T, Wareham N, Bingham S, Luben R, Welch A, Day N. Association of hemoglobin A1C with cardiovascular disease and mortality in adults: The European Prospective Investigation into Cancer in Norfolk. Ann Intern Med. 2004;141(6):413-420.

25. Malmberg K, Ryden L. Myocardial infarction in patients with diabetes mellitus. Eur Heart J. 1988;9(3):259-264.

26. Meier JJ, Deifuss S, Klamann A, Launhardt V, Schmiegel WH, Nauck MA. Plasma glucose at hospital admission and previous metabolic control determine myocardial infarct size and survival in patients with and without type 2 diabetes: The Langendreer Myocardial Infarction and Blood Glucose in Diabetic Patients Assessment (LAMBDA). Diabetes Care. 2005;28(10):2551-2553.

27. Mehta SR, Yusuf S, Diaz R, et al. Effect of glucose-insulin-potassium infusion on mortality in patients with acute ST-segment elevation myocardial infarction: The CREATE-ECLA randomized controlled trial. JAMA. 2005;293(4):437-446.

28. Bolk J, van der Ploeg TJ, Cornel JH, Arnold AER, Sepers J, Umans VAWM. Impaired glucose metabolism predicts mortality after a myocardial infarction. Int J Cardiol. 2001;79(2-3):207-214.

29. Furnary AP, Gao G, Grunkemeier GL, et al. Continuous insulin infusion reduces mortality in patients with diabetes undergoing coronary artery bypass grafting. J Thorac Cardiovasc Surg. 2003;125(5):1007-1021.
30. Capes SE, Hunt D, Malmberg K, Gerstein HC. Stress hyperglycaemia and increased risk of death after myocardial infarction in patients with and without diabetes: A systematic overview. Lancet. 2000;355(9206): 773-778.

31. Lemesle G, Bonello L, de Labriolle A, et al. Prognostic value of hemoglobin A1C levels in patients with diabetes mellitus undergoing percutaneous coronary intervention with stent implantation. Am J Cardiol. 2009;104(1):41-45.

32. Aronson D, Hammerman H, Suleiman M, Markiewicz W. Usefulness of changes in fasting glucose during hospitalization to predict long-term mortality in patients with acute myocardial infarction. Am J Cardiol. 2009;104(8):1013-1017.

33. Ingels C, Debaveye Y, Milants I, et al. Strict blood glucose control with insulin during intensive care after cardiac surgery: Impact on 4-years survival, dependency on medical care, and quality-of-life. Eur Heart J. 2006;27(22):2716-2724.

34. Malmberg K, Rydén L, Efendic S, et al. Randomized trial of insulinglucose infusion followed by subcutaneous insulin treatment in diabetic patients with acute myocardial infarction (DIGAMI study): Effects on mortality at 1 year. J Am Coll Cardiol. 1995;26(1):57-65.

35. Malmberg K; for the DIGAMI (Diabetes Mellitus Insulin Glucose Infusion in Acute Myocardial Infarction) Study Group. Prospective randomised study of intensive insulin treatment on long term survival after acute myocardial infarction in patients with diabetes mellitus. BMJ. 1997;314(7093):1512-1515.

36. Malmberg K, Ryden L, Wedel H, et al. Intense metabolic control by means of insulin in patients with diabetes mellitus and acute myocardial infarction (DIGAMI 2): Effects on mortality and morbidity. Eur Heart J. 2005;26(7):650-661.

37. Magee MF. Insulin therapy for intensive glycemic control in hospital patients. Hosp Physician. 2006;38:17-28.

38. Furnary AP, Wu Y. Clinical effects of hyperglycemia in the cardiac surgery population: The Portland Diabetic Project. Endocr Pract. 2006; 12 Suppl 3:22-26.

39. The Action to Control Cardiovascular Risk in Diabetes Study Group. Effects of intensive glucose lowering in type 2 diabetes. $N$ Engl J Med. 2008;358(24):2545-2559.

40. Duckworth W, Abraira C, Moritz T, et al. Glucose control and vascular complications in veterans with type 2 diabetes. $N$ Engl J Med. 2009; 360(2):129-139.

41. The ADVANCE Collaborative Group. Intensive blood glucose control and vascular outcomes in patients with type 2 diabetes. $N$ Engl J Med. 2008;358(24):2560-2572.

42. Skyler JS, Bergenstal R, Bonow RO, et al. Intensive glycemic control and the prevention of cardiovascular events: Implications of the ACCORD, ADVANCE, and VA diabetes trials: A position statement of the American Diabetes Association and a Scientific Statement of the American College of Cardiology Foundation and the American Heart Association. Circulation. 2009;119(2):351-357.

43. Kerr M. Intensive glycemic control not directly linked to excess cardiovascular risk. Heartwire. 2009 Jun 10. Available from http://www.theheart.org/article/978067.do. Accessed 2010 Sep 20.

44. Currie CJ, Peters JR, Tynan A, et al. Survival as a function of $\mathrm{HbA}_{1 \mathrm{c}}$ in people with type 2 diabetes: A retrospective cohort study. Lancet. 2010;375(9713):481-489.

45. Balkau B, Simon D. Survival in people with type 2 diabetes as a function of $\mathrm{HbA}_{1 \mathrm{c}}$. Lancet. 2010;375(9713):438-440.

46. US National Institutes of Health. ClinicalTrials.gov Web site. The ORIGIN Trial (outcome reduction with initial glargine intervention). Available from: http://clinicaltrials.gov/ct2/results?term=NCT00069784. Accessed 2010 Sep 20.

47. US National Institutes of Health. ClinicalTrials.gov Web site. Impact of tight glycaemic control in acute myocardial infarction. Available from: http:// clinicaltrials.gov/ct2/results?term=NCT00237471. Accessed 2010 Sep 20.

48. Preiser JC, Devos P, Ruiz-Santana S, et al. A prospective randomised multi-centre controlled trial on tight glucose control by intensive insulin therapy in adult intensive care units: The Glucontrol study. Intensive Care Med. 2009;35(10):1738-1748. 
49. Brunkhorst FM, Engel C, Bloos F, et al. Intensive insulin therapy and pentastarch resuscitation in severe sepsis. $N$ Engl J Med. 2008;358(2): 125-139.

50. Lipshutz AK, Gropper MA. Perioperative glycemic control: An evidence-based review. Anesthesiology. 2009;110(2):408-421.

51. Arabi YM, Tamim HM, Rishu AH. Hypoglycemia with intensive insulin therapy in critically ill patients: Predisposing factors and association with mortality. Crit Care Med. 2009;37(9):2536-2544.

52. Yu WK, Li WQ, Wang XD, et al. [Influence and mechanism of a tight control of blood glucose by intensive insulin therapy on human sepsis]. Zhonghua Wai Ke Za Zhi. 2005;43(1):29-32.

53. Henderson WR, Dhingra VK, Chittock DR, et al. Survival Using Glucose Algorithm Regulation (SUGAR) Trial_-Pilot Data. Presented at: the American Thoracic Society 2005 International Congress; 2005 May 22; San Diego, CA. Abstract A23.

54. Mitchell I, Knight E, Gissane J, et al. A phase II randomised controlled trial of intensive insulin therapy in general intensive care patients. Crit Care Resusc. 2006;8(4):289-293.

55. Wang LC, Lei S, Wu YC, et al. [Intensive insulin therapy in critically ill patients]. Zhongguo Wei Zhong Bing Ji Jiu Yi Xue. 2006;18(12): $748-750$.

56. Azevedo JR, de Araujo LO, Azevedo RP. Intensive insulin therapy versus glycemic control in critically ill patients: a prospective controlled trial. Crit Care. 2007:11(suppl 3):S34.

57. McMullin J, Brozek J, McDonald E, et al. Lowering of glucose in critical care: a randomized pilot trial. J Crit Care. 2007;22(2):112-118.

58. Devos P, Preiser J, Melot C. Impact of tight glucose control by intensive insulin therapy on ICU mortality and the rate of hypoglycaemia: final results of the GLUCONTROL study. Intensive Care Med. 2007;33:S189.

59. Iapichino G, Albicini M, Umbrello M, et al. Tight glycemic control does not affect asymmetric-dimethylarginine in septic patients. Intensive Care Med. 2008;34(10):1843-1850.

60. He ZY, Li N, Xing J. Effect of intensive insulin therapy on shortterm outcome in critically ill patients. Chinese J Clin Nutr. 2008;16: 220-222.

61. Zhang R, He W, Li T. Evaluation of optimal goal of glucose control in critically ill patients. Chinese J Clin Nutr. 2008;16:204-208.

62. De La Rosa Gdel C, Donado JH, Restrepo AH, et al. Strict glycaemic control in patients hospitalised in a mixed medical and surgical intensive care unit: a randomised clinical trial. Crit Care. 2008;12(5):R120.

63. Arabi YM, Dabbagh OC, Tamim HM, et al. Intensive versus conventional insulin therapy: a randomized controlled trial in medical and surgical critically ill patients. Crit Care Med. 2008;36(12):3190-3197.

64. Mackenzie IM, Ercole A, Ingle S. Glycaemic control and outcome in general intensive care: The east anglian GLYCOGENIC study. $\mathrm{Br} J$ Intensive Care. 2008;18:121-126.

65. Bland DK, Fankhanel Y, Langford E, et al. Intensive versus modified conventional control of blood glucose level in medical intensive care patients: a pilot study. Am J Crit Care. 2005;14(5):370-376.

66. Walters MR, Weir CJ, Lees KR. A randomised, controlled pilot study to investigate the potential benefit of intervention with insulin in hyperglycaemic acute ischaemic stroke patients. Cerebrovasc Dis. 2006;22(2-3):116-122.

67. Farah R, Samokhvalov A, Zviebel F, Makhoul N. Insulin therapy of hyperglycemia in intensive care. Isr Med Assoc J. 2007;9(3): 140-142.

68. Oksanen T, Skrifvars MB, Varpula T, et al. Strict versus moderate glucose control after resuscitation from ventricular fibrillation. Intensive Care Med. 2007;33(12):2093-2100.

69. Bruno A, Kent TA, Coull BM, et al. Treatment of Hyperglycemia In Ischemic Stroke (THIS). A Randomized Pilot Trial. Stroke. 2008;39(2): 384-389.

70. Grey NJ, Perdrizet GA. Reduction of nosocomial infections in the surgical intensive-care unit by strict glycemic control. Endocr Pract. 2004;10(suppl 2):46-52.
71. Bilotta F, Spinelli A, Giovannini F, Doronzio A, Delfini R, Rosa G. The effect of intensive insulin therapy on infection rate, vasospasm, neurologic outcome, and mortality in neurointensive care unit after intracranial aneurysm clipping in patients with acute subarachnoid hemorrhage: a randomized prospective pilot trial. $J$ Neurosurg Anesthesiol. 2007;19(3):156-160.

72. He W, Zhang TY, Zhou H, et al. [Impact of intensive insulin therapy on surgical critically ill patients]. Zhonghua Wai Ke Za Zhi. 2007;45(15):1052-1054.

73. Bilotta F, Caramia R, Cernak I, et al. Intensive insulin therapy after severe traumatic brain injury: a randomized clinical trial. Neurocrit Care. 2008;9(2):159-166.

74. Vlasselaers D, Milants I, Desmet L, et al. Intensive insulin therapy for patients in paediatric intensive care: a prospective, randomised controlled study. Lancet. 2009;373(9663):547-556.

75. Yang M, Guo Q, Zhang X, et al. Intensive insulin therapy on infection rate, days in NICU, in-hospital mortality and neurological outcome in severe traumatic brain injury patients: a randomized controlled trial. Int J Nurs Stud. 2009;46(6):753-758.

76. Quinn TJ, Dawson J, Walters MR. Sugar and stroke: Cerebrovascular disease and blood glucose control. Cardiovasc Ther. 2010 May 13. [Epub ahead of print].

77. Baird TA, Parsons MW, Phanh T, et al. Persistent poststroke hyperglycemia is independently associated with infarct expansion and worse clinical outcome. Stroke. 2003;34(9):2208-2214.

78. Capes SE, Hunt D, Malmberg K, Pathak P, Gerstein HC. Stress hyperglycemia and prognosis of stroke in nondiabetic and diabetic patients: a systematic overview. Stroke. 2001;32(10):2426-2432.

79. Gray CS, Hildreth AJ, Sandercock PA, et al. Glucose-potassiuminsulin infusions in the management of post-stroke hyperglycaemia: the UK Glucose Insulin in Stroke Trial (GIST-UK). Lancet Neurol. 2007;6(5):397-406.

80. Ali NA, O’Brien JM, Jr., Dungan K, et al. Glucose variability and mortality in patients with sepsis. Crit Care Med. 2008;36(8):2316-2321.

81. Egi M, Bellomo R, Stachowski E, French CJ, Hart G. Variability of blood glucose concentration and short-term mortality in critically ill patients. Anesthesiol. 2006;105(2):244-252.

82. Krinsley JS. Glycemic variability: a strong independent predictor of mortality in critically ill patients. Crit Care Med. 2008;36(11): 3008-3013.

83. Dossett LA, Cao H, Mowery NT, Dortch MJ, Morris JM, Jr., May AK. Blood glucose variability is associated with mortality in the surgical intensive care unit. Am Surg. 2008;74(8):679-685.

84. Al-Dorzi HM, Tamim HM, Arabi YM. Glycaemic fluctuation predicts mortality in critically ill patients. Anaesth Intensive Care. 2010;38(4):695-702.

85. American Diabetes Association. Standards of medical care in diabetes - 2007. Diabetes Care. 2007;30(suppl 1):S4-S41.

86. Bode BW, Braithwaite SS, Steed RD, Davidson PC. Intravenous insulin infusion therapy: indications, methods, and transition to subcutaneous insulin therapy. Endocr Pract. 2004;10(suppl 2):71-80.

87. Goldberg PA, Siegel MD, Sherwin RS, et al. Implementation of a safe and effective insulin infusion protocol in a medical intensive care unit. Diabetes Care. 2004;27(2):461-467.

88. Krinsley JS. Effect of an intensive glucose management protocol on the mortality of critically ill adult patients. Mayo Clin Proc. 2004;79(8): 992-1000

89. American Diabetes Association. Standards of medical care in diabetes - 2008. Diabetes Care. 2008;31(suppl 1):S12-S54.

90. Schultz MJ, Royakkers AA, Levi M, Moeniralam HS, Spronk PE. Intensive insulin therapy in intensive care: an example of the struggle to implement evidence-based medicine. PLoS Med. 2006;3(12):e456.

91. Goldberg PA, Sakharova OV, Barrett PW, et al. Improving glycemic control in the cardiothoracic intensive care unit: clinical experience in two hospital settings. J Cardiothorac Vasc Anesth. 2004;18(6): 690-697. 
92. Quinn JA, Snyder SL, Berghoff JL, Colombo CS, Jacobi J. A practical approach to hyperglycemia management in the intensive care unit: evaluation of an intensive insulin infusion protocol. Pharmacotherapy. 2006;26(10):1410-1420.

93. Buchs AE, Bloemers R, Shiloah E, et al. Feasibility and safety of multiple daily insulin injections in general medicine wards. Eur J Intern Med. 2009;20(4):435-439.

94. Blaha J, Kopecky P, Matias M, et al. Comparison of three protocols for tight glycemic control in cardiac surgery patients. Diabetes Care. 2009;32(5):757-761.

95. Avanzini F, Marelli G, Donzelli W, et al. Hyperglycemia during acute coronary syndrome: a nurse-managed insulin infusion protocol for stricter and safer control. Eur J Cardiovasc Nurs. 2009;8(3):182-189.
96. Yeldandi RR, Lurie A, Baldwin D. Comparison of once-daily glargine insulin with twice-daily NPH/regular insulin for control of hyperglycemia in inpatients after cardiovascular surgery. Diabetes Technol Ther. 2006;8(6):609-616.

97. Umpierrez GE, Smiley D, Zisman A, et al. Randomized study of basalbolus insulin therapy in the inpatient management of patients with type 2 diabetes (RABBIT 2 trial). Diabetes Care. 2007;30(9):2181-2186.

98. Cook CB, Seifert KM, Hull BP, et al. Inpatient to outpatient transfer of diabetes care: planing for an effective hospital discharge. Endocr Pract. 2009;15(3):263-269.

99. Lavernia F. Treating hyperglycemia and diabetes with insulin therapy: transition from inpatient to outpatient care. Medscape J Med. 2008;10:216-233

\section{Publish your work in this journal}

Vascular Health and Risk Management is an international, peerreviewed journal of therapeutics and risk management, focusing on concise rapid reporting of clinical studies on the processes involved in the maintenance of vascular health; the monitoring, prevention and treatment of vascular disease and its sequelae; and the involvement of

\section{Dovepress}

metabolic disorders, particularly diabetes. This journal is indexed on PubMed Central and MedLine. The manuscript management system is completely online and includes a very quick and fair peer-review system, which is all easy to use. Visit http://www.dovepress.com/ testimonials.php to read real quotes from published authors.

Submit your manuscript here: http://www.dovepress.com/vascular-health-and-risk-management-journal 